\title{
Strain on the Framework of a Mandibular Free-End Saddle Partial Denture Under Load*
}

\author{
R. G. CRAIG and F. A. PEYTON, School of Dentistry, University of \\ Michigan, Ann Arbor, Michigan 48104
}

\begin{abstract}
Summary
Strains were determined in a typical mandibular partial denture metal framework of the free-end saddle type. The overall strain patterns were determined using a brittle lacquer coating technique and loads were applied vertically to positions on the saddles. The results from the coating study indicated the area of high strain were just anterior and posterior to the finishing lines. Strain gages were attached to positions anterior and posterior to the finishing lines and the strain was measured at these positions when the saddles were loaded either at a posterior or anterior position at intervals of $2 \mathrm{lb} u p$ to $20 \mathrm{lb}$. When a static load of $20 \mathrm{lb}$ was applied to the posterior of the left saddle a strain of $750 \mu \mathrm{in}$./in. was obtained, which for the alloy used was equivalent to a stress of $22,200 \mathrm{lb} / \mathrm{in} .{ }^{2}$. The amount of strain observed was a function of the length of the saddle, the bulk of the framework, the relation of the site of the gage to the position of loading, the presence of an indirect retainer, and the position of the fulcrum for the loading conditions used.
\end{abstract}

The maximum forces and the forces during mastication have been measured by a number of investigators, ${ }^{1-5}$ using a variety of prosthetic appliances. It has been concluded that the maximum force on a single tooth in a partial denture is between 20 and $25 \mathrm{lb}$, and that the normal chewing forces on a single tooth may be up to $8 \mathrm{lb}$.

Forces on the teeth of these partial dentures transfer stress to the metal framework and of course to the supporting teeth. Frechette ${ }^{6}$ has determined the magnitude of forces on the abutment teeth of a model, as well as the horizontal movement, using partial dentures of different designs. He has observed the loading and movement of teeth to be a function of the number and location of the rests, the

* Paper presented at 44th General Meeting of the International Association for Dental Research, March 25, 1966, Miami, Florida. 
contours and rigidity of the lingual connectors, and the extension of the base. Kaires ${ }^{7,8}$ has studied the effect of the design of the partial denture on the unilateral and bilateral distribution of the force on the abutment teeth and related structures. He has observed that a ring clasp, reinforced with a buccal arm, reduced its flexibility and concluded a rigid lingual bar and maximum coverage of the denturebearing areas were desirable.

Bates ${ }^{9}$ recently has reported some preliminary measurements of the functional strain in chromium-cobalt partial dentures. He has used brittle lacquer coatings to establish positions to attach silicon transducer strain gages and has recorded the output on an oscilloscope. Strains on the lingual bar during function were as much as 1,000 $\mu \mathrm{in} . / \mathrm{in}$. representing a stress of $30,000 \mathrm{lb} / \mathrm{in} .{ }^{2}$.

The purpose of this study was to measure the direction and concentration of strain on the metal framework of a typical mandibular partial denture having bilateral free-end saddles, using brittle lacquer coatings. After the establishment of areas of high stress the ultimate purpose was to quantitatively measure the surface strain with strain gages.

\section{Materials and Methods}

A typical mandibular model was chosen which is shown in Figure $1 A$ and $B$. The patient was missing the left second bicuspid, and the left and right first and second molars. The model was constructed of poly (methyl methacrylate) and $1.5 \mathrm{~mm}$ of silicone rubber was used to support the base and simulate the soft tissues. The lingual bar was of the half-round shape with a thickness (radius) of approximately $2 \mathrm{~mm}$ and a depth of approximately $4 \mathrm{~mm}$. Buccal clasps of wrought wire (PGP) were used on the left first bicuspid and right second bicuspid. Rests were provided on the left and right cuspid and first bicuspids. Cast lingual clasps were used on the right second bicuspid and on the left first bicuspid where it was tied to the rest. The partial denture framework was cast from a low-fusing chromium nickel alloy (Ticonium 100, Ticonium Division of CMP Industries, Albany, New York) using standard recommended laboratory procedures.

Lacquer coatings (Stresscoat 1204, Magnaflux Corporation, Chicago, Illinois) were sprayed on the metal framework on all surfaces not contacting the model. After the lacquer was no longer 


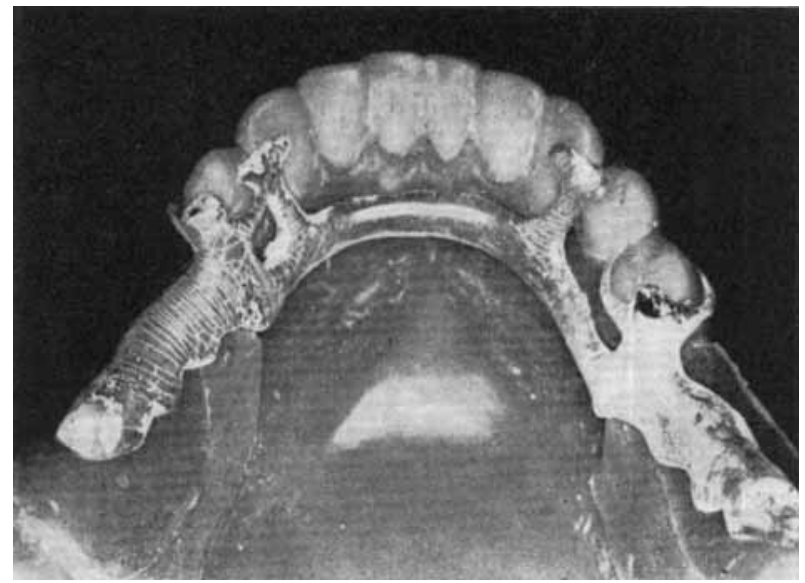

(A)

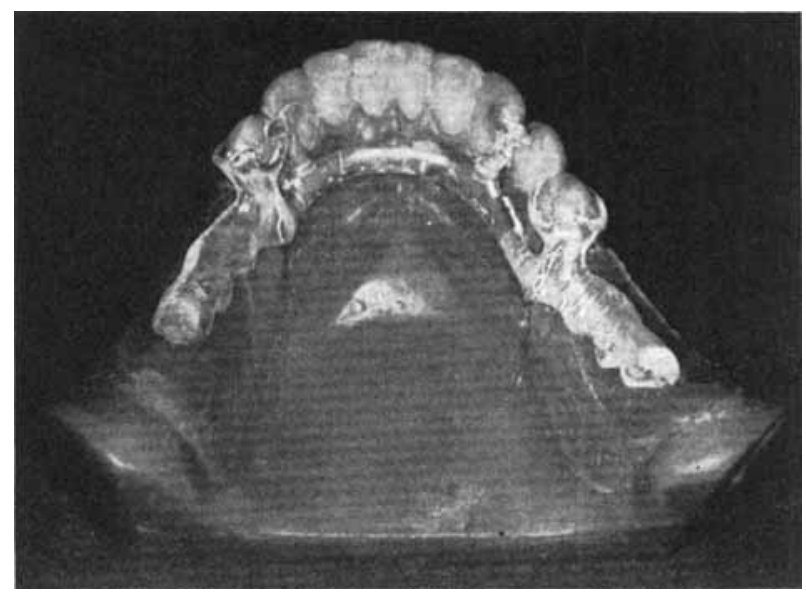

(B)

Fig. 1. Brittle lacquer crack patterns resulting from loading at $(A)$ the left posterior saddle position, and $(B)$ the right anterior saddle position.

tacky the partial denture was placed on the model and the lacquer was cured at $40^{\circ} \mathrm{C}$ for $16 \mathrm{hr}$. The model was allowed to cool $2 \mathrm{hr}$ at room temperature of $22^{\circ} \mathrm{C}$ and $40 \%$ humidity. Test bars sprayed under these conditions gave sensitivities for the lacquer of 500 to $580 \mu \mathrm{in} . /$ in. (MII). 
Static loads were applied to the areas of interest in increments of 2 $\mathrm{lb}$ up to a maximum of $20 \mathrm{lb}$. Cracks in the lacquer coatings were observed visually using light reflected at low angles. The positions of the cracks and the corresponding loads were recorded. In several instances after loading to $20 \mathrm{lb}$ the surface of the lacquer was painted with a liquid detergent, the load removed, the excess detergent wiped away, and the model was sprayed with talc. (Statiflux, Magnaflux Corporation, Chicago, Illinois). This procedure allowed the cracks to be photographed easily as seen in Figure $1 A$ and $B$.

Foil SR-4 strain gages (FA-03-12-L Baldwin, Lima-Hamilton Corporation, Waltham, Mass.) were attached with a cyanoacrylate adhesive (Eastman 910, Eastman Chemical Products Inc., Kingsport, Tenn.) to the surface of the chromium-nickel partial denture and the strain recorded using a strain gage amplifier and meter (Daytronic $300 \mathrm{CP}$ with a Type 80 plug-in unit, Daytronic Corp., Dayton, Ohio). The gage was used as a single active arm and a dummy strain gage (FA-O3-12-L Baldwin, Lima-Hamilton Corporation, Waltham, Mass.) was used for temperature compensation. The amplifier unit contained internal calibration standards such that the strain was read off the meter directly in MII. The static load was applied vertically to various positions on the saddle using a $1 / 8$-in. diameter steel ball and the strain recorded for various positions of the strain gage. For each condition the plot of strain versus load was determined from five individual sets of data. The strain readings for the same loading conditions could be reproduced within $\pm 5 \%$ of the seale reading.

\section{Results}

The crack pattern obtained from the brittle lacquer coating using the sprayed powder technique is shown in Figure $1 A$ for loading of the partial denture on the posterior portion of the left saddle. The direction of the cracks on saddle area were in a buccal-lingual direction, which indicated the principal tensile strain was $90^{\circ}$ to this direction or followed the crest of the ridge. Two families of cracks were obtained in the central portion the lingual bar at about $45^{\circ}$ to the vertical indicating a biaxial stress field. The direction of the principal stresses shifted from a horizontal direction at the abutment teeth to $45^{\circ}$ in both directions from horizontal near the center of the lingual bar. The cracks in the lacquer on the minor connectors to the rests were in a horizontal direction, and thus the principal 


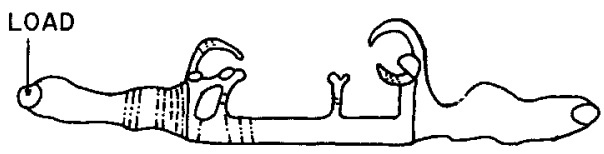

(A)

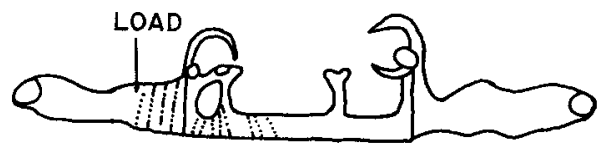

(B)

Fig. 2. Brittle lacquer crack patterns at various loads when loaded at $(A)$ the left posterior saddle position, and $(B)$ the left anterior saddle position: (- -

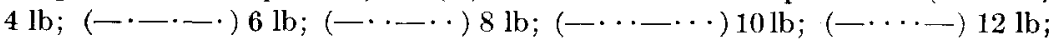
$(-\cdots \cdots-) 16 \mathrm{lb} ;(\cdots \cdots) 18 \mathrm{lb}$.

stress was in the vertical direction. The lacquer cracks on the clasps showed the principal stress to be in the direction of the long axes.

The crack pattern in Figure $1 B$ was a result of loading the partial denture in the first molar region of the right saddle area. Similar crack patterns were observed but the extent of cracking was less at $20 \mathrm{lb}$ load than when it was loaded at the posterior position on the left side. Stresses were observed on the minor connectors and clasps but not on the left area.

Figure $2 A$ and $B$ show sketches of the crack patterns obtained on the saddles and lingual bar when loaded at the posterior and anterior positions of the left saddle. The principal point of interest is that the cracks form at low loads near the finishing line and as the loads were increased cracks appeared more posterior in the saddle area and more anterior on the lingual bar.

Based on data from the brittle lacquer coatings the area of high stress concentration would be just posterior and anterior to the finishing line. Foil strain gages were cemented to the surface of the metal framework just posterior and anterior to the finishing lines and loads were applied at the positions indicated in Figures 3 through 6 . Loads from zero to $20 \mathrm{lb}$ were applied at intervals of $2 \mathrm{lb}$ and the strain in MII was measured at the four positions of the strain gage indicated in the figures. 
The strain at the four positions when the load was applied at the left posterior saddle area is given in Figure 3 . At a load of $20 \mathrm{lb}$ the strain gage at position 1, posterior to the left finishing line, recorded the highest value of 740 MII. This value can be converted to surface stress by multiplying by the elastic modulus of $30 \times 10^{6} \mathrm{lb} / \mathrm{in}^{2}$,

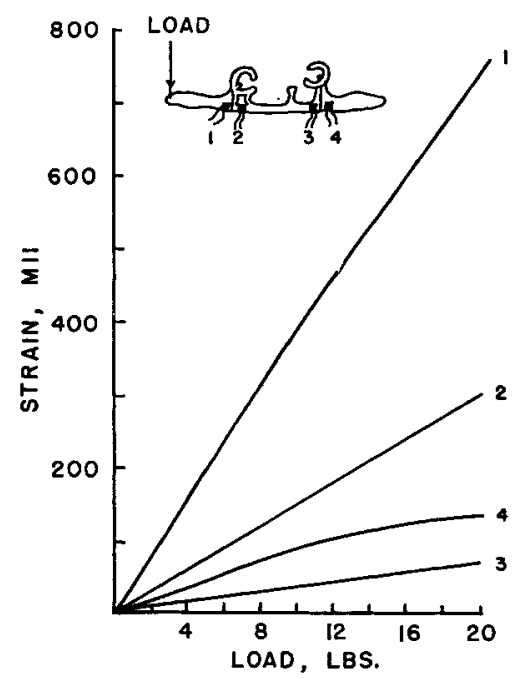

Fig. 3. Strain versus load for gage position anterior and posterior to the finishing lines with static vertical loads applied to the posterior position of the left saddle.

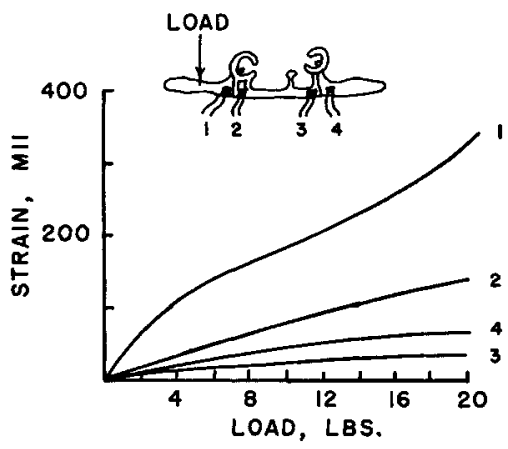

Fig. 4. Strain versus load for gage positions 1 through 4 when static vertical loads were applied to the anterior position of the left saddle. 


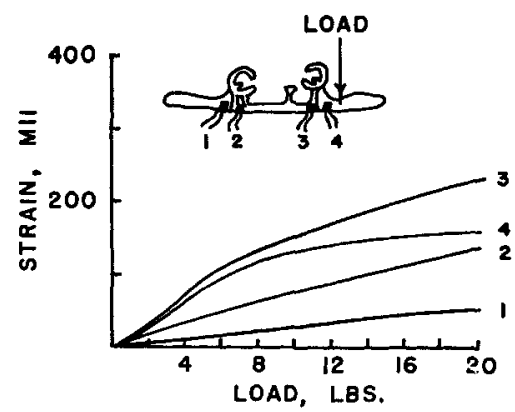

Fig. 5. Strain versus load for gage position 1 through 4 when static vertical loads were applied to the anterior position of the right saddle.

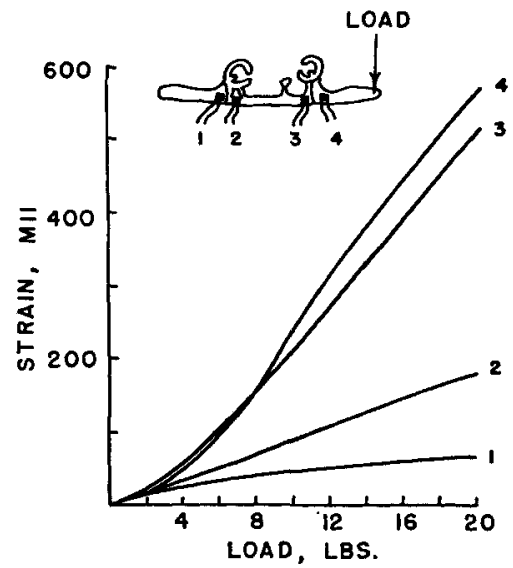

Fig. 6. Strain versus load for gage position 1 through 4 when static vertical loads were applied to the posterior position of the right saddle.

which gives a value of $22,200 \mathrm{lb} / \mathrm{in} .^{2}$. The strain just anterior to the finishing line, was lower than the posterior position and is shown in Figure 3 as curve 2 . The strain at $20 \mathrm{lb}$ was 310 MII. Smaller strains of 75 and $130 \mathrm{MII}$ were observed at $20 \mathrm{lb}$ load for positions 3 and 4 , just anterior and posterior to the right finishing line.

The strain versus load curves from the four gage positions when the loading was on the anterior portion of the left saddle are shown in Figure 4. The order of the strain at any specified load is the same as for loading the partial denture at the posterior portion of the 
saddle but the magnitudes of the strains are lower. For example, the strain at gage position 1 was $350 \mathrm{MII}$ with the load in the anterior position compared with 740 MII when the load of $20 \mathrm{lb}$ was applied at the posterior position.

Strain-load curves are shown in Figure 5 for the various strain gages when the load was applied to the anterior portion of the right saddle. These curves are similar to those obtained when the comparable position on the left side was loaded but of course the order of the curves was different. The decreasing order of strain when loading at $20 \mathrm{lb}$ was at gage position $3,4,2$, and 1 . The maximum strain recorded for this load position was $230 \mathrm{MII}$ for strain gage 3 at $20 \mathrm{lb}$ load.

The strain-load curves obtained when the load was applied to the posterior position of the right saddle are presented in Figure 6. Strain values were higher, 520 to $570 \mathrm{MII}$ at $20 \mathrm{lb}$ load, for strain gages 3 and 4 which were attached to the right side of the bridge and were lower, 60 to $170 \mathrm{MII}$, for the gages on the left side of the partial denture.

\section{Discussion}

Linear regression analysis and correlation of strain and load were determined for curves representing the same position of loading. In critical situations, curves 3 and 4 in Figures 4, 5, and 6 the significance of the slopes were tested by a $t$-test.

The strain versus load curves for the 4 positions of loading are plotted in Figures 7 through 10 using the least-square method. The values of $a$ and $b$ for the slope-intercept equation, $y=a+b x$, are given for the lines as well as the standard error, $S_{x \cdot y}$, and the angle $\theta$ between the line and the $x$ (load) axis. The calculated $r$ values for each line are shown; 13 of the lines had $r$ values of 0.99 or better and the remaining 3 lines had values between 0.96 and 0.98 . The lines showed regression and nearly perfect correlation $(r=1)$ as would be expected from the interdependency of strain and load. Many of the lines could be drawn through the origin and where they did not pass through zero it was probably a result of accuracy of the data at low values of strain.

On the basis of these calculations it is possible to estimate the strain at loads other than the experimental values taken at 2-lb intervals. 


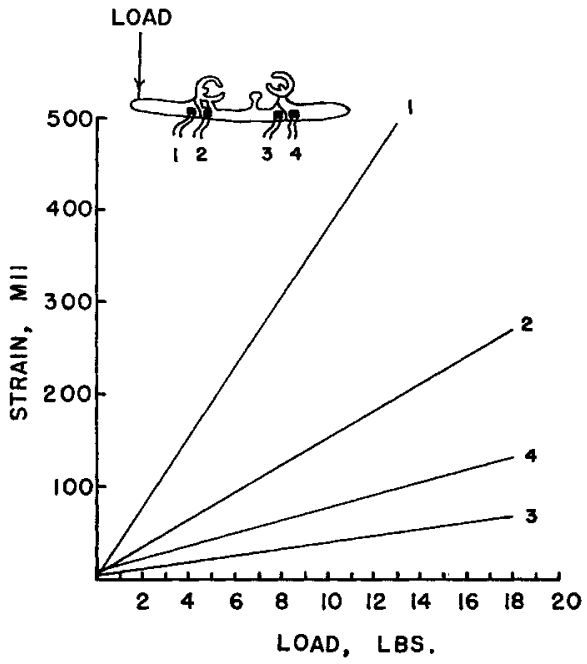

Fig. 7. Linear regression and correlation of strain versus load when the load was applied to the anterior position of the left saddle: $a_{1}=8.7, b_{1}=37.3$, $r=0.99, S_{y \cdot x}=13, \theta_{1}=56^{\circ} ; a_{2}=5.9, b_{2}=14.2, r=0.99_{8}, S_{y \cdot x}=3.35$, $\theta_{2}=34^{\circ} ; a_{3}=3.8, b_{3}=3.25, r=0.99, S_{y \cdot x}=2.25, \theta_{3}=8^{\circ} ; a_{4}=11.0$, $b_{4}=6.84, r=0.99, S_{y . x}=10.3, \theta_{4}=13^{\circ}$.

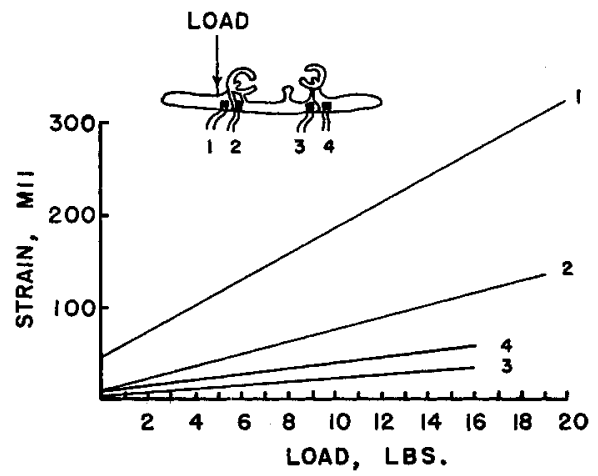

Fig. 8. Linear regression and correlation of strain versus load when the load was applied to the posterior position of the left saddle: $a_{1}=45, b_{1}=13.97$, $r=0.99, S_{y . x}=25.5, \theta_{1}=29^{\circ} ; a_{2}=10.5, b_{2}=6.6, r=0.99, S_{y . x}=5.88$, $\theta_{2}=14.5^{\circ} ; a_{3}=3.5, b_{3}=1.82, r=0.96, S_{y . x}=3.48, \theta_{3}=4.5^{\circ} ; a_{4}=8.8$, $b_{4}=2.94, r=0.98, S_{y . x}=7.3, \theta_{4}=10^{\circ}$. 


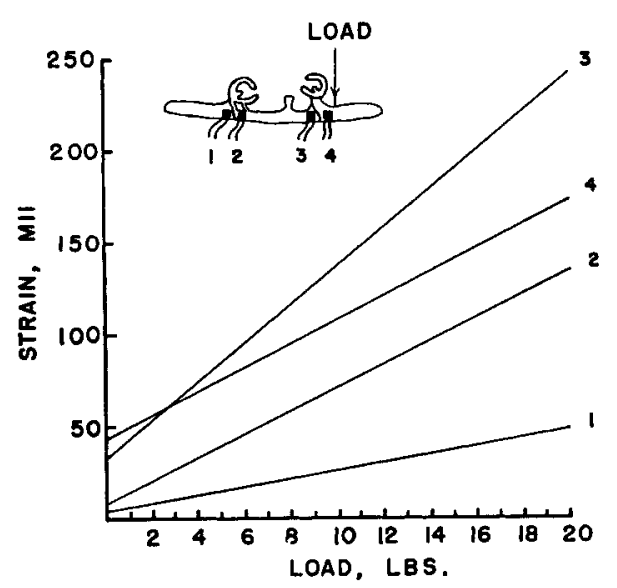

Fig. 9. Linear regression and correlation of strain versus load when the load was applied to the anterior position of the right saddle: $a_{1}=6.2, b_{1}=2.26$, $r=0.99, S_{y . x}=4.5, \theta_{1}=10^{\circ} ; a_{2}=9.3, b_{2}=6.32, r=0.99, S_{y . x}=6.1$, $\theta_{2}=27^{\circ} ; a_{3}=32.2, b_{3}=10.33, r=0.99, S_{y \cdot x}=25.2, \theta_{3}=39^{\circ} ; a_{4}=44.3$, $b_{4}=6.47, r=0.97, S_{y . x}=29.6, \theta_{4}=26^{\circ}$.

The difference between curves 3 and 4 in Figure 8 was tested using the $t$-test to test the difference between the $b$ values (slopes) in the equations $y_{3}=3.5+1.82 x_{3}$ and $y_{4}=8.8+2.94 x_{4}$. A two-tailed test was used and the $t_{16}$ value of 2.50 resulted in rejection of the null hypothesis that the two slopes were the same at the $5 \%$ confidence level (critical $t=2.12$ ).

A similar test on the difference in the slopes was run on curves 3 and 4 in Figures 9 and 10 . Experimental $t_{16}$ values of 1.81 and 1.43 were obtained which led to the acceptance of the null hypothesis that the slopes of curves 3 and 4 in each figure were not different at the $5 \%$ confidence level (critical $t=2.12$ ). Other than these exceptions the four curves in each of Figures 7 through 10 were significantly different.

The brittle lacquer studies illustrated that loads applied to the freeend saddle of a bilateral partial denture caused high stress concentrations to occur in the area of the finishing line on the same side as the application of load and it would be anticipated that if fracture occurred in this type of partial denture it would likely take place in this area. The saddle functions as a cantilever and these stresses 


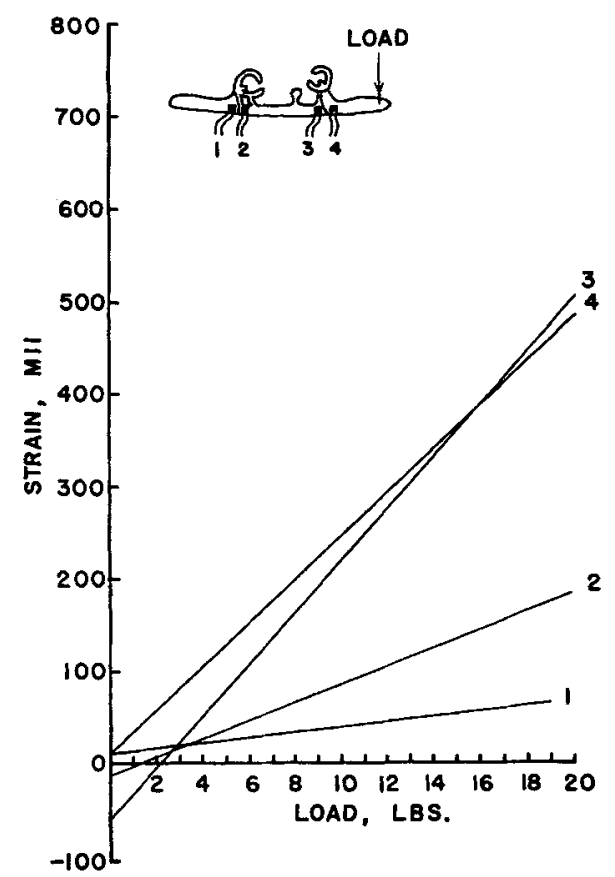

Fig. 10. Linear regression and correlation of strain versus load when the load was applied to the posterior position of the right saddle. $a_{1}=14, b_{1}=2.65$, $r=0.99, S_{y . x}=8.29, \theta_{1}=6^{\circ} ; a_{2}=-12.8, b_{2}=9.73, r=0.99, S_{y . x}=7.26$, $\theta_{2}=22^{\circ} ; a_{3}=-54.5, b_{3}=28, r=0.99, S_{y . x}=29.3, \theta_{3}=49^{\circ} ; a_{4}=17.0$, $b_{4}=32.4, r=0.99, S_{y . x}=45.7, \theta_{4}=43^{\circ}$.

appear first at the abutment area and proceed in both an anterior and posterior direction from this position because the position of the fulcrum is the abutment. Higher stresses were observed from loading the left saddle when the strain gages were posterior to the finishing line, which is a reflection of the length of the saddle, since three teeth were missing on the left and only two on the right side. Less stress was observed on the left than on the right side when the strain gage was just anterior to the abutment, and it is proposed that this effect is a result of the greater bulk of the partial denture on the left side where the vertical minor connector and clasp are connected to the lingual clasp.

When the load was applied to the left saddle the order of decreasing strain was (1) posterior to the left finishing line, (2) anterior to the 
left finishing line, (3) posterior to the right finishing line, and (4) anterior to the right finishing line. More strain at gage position 4 than 3 probably results from the effect of the indirect retainer on the cuspid and bicuspid which overshadowed the fact that position 4 was further from the site of loading.

When the load was applied to the right saddle area lower strains were observed because the length of the saddle was less on the right side. Gage position 3 and 4 had strain values which were not statistically different which lends support to the abutment being the fulcrum when a compressive load is applied to the right saddle area. Gage position 1 showed less strain than position 2 which is a reflection of the distance of the position from the site of loading.

The authors express their appreciation of M. Kamal El Ebrashi for his assistance in the interpretation of the data.

This investigation was supported in whole by U.S.P.H.S. Research Grant DE-01817 from the National Institute for Dental Research, National Institutes of Health, Bethesda, Maryland.

\section{References}

1. A. H. Howell and R. S. Manly, J. Dent. Res., 27, 705 (1948).

2. A. H. Howell and F. Brudevold, J. Dent. Res., 29, 133 (1950).

3. A. Yurkatas and W. A. Curby, J. Prost. Dent., 3, 82 (1953).

4. D. J. Anderson, J. Dent. Res., 35, 664, 671 (1956).

5. D. J. Anderson and D. C. A. Picton, J. Dent. Res., 37, 312 (1958).

6. A. R. Frechette, J. Prost. Dent., 6, 195 (1956).

7. A. K. Kaires, J. Prost. Dent., 6, 373 (1956).

8. A. K. Kaires, J. Prost. Dent., 6, 526 (1956).

9. J. F. Bates, Brit. Dent. J., 120, 79 (1966).

Received January 16, 1967 